\title{
Behavioral Stress Enhances Hippocampal CA1 Long-Term Depression through the Blockade of the Glutamate Uptake
}

\author{
Chih-Hao Yang, ${ }^{1,2}$ Chiung-Chun Huang, ${ }^{1}$ and Kuei-Sen $\mathrm{Hsu}^{1,2}$ \\ ${ }^{1}$ Department of Pharmacology and ${ }^{2}$ Institute of Basic Medical Sciences, College of Medicine, National Cheng Kung University, Tainan 701, Taiwan
}

\begin{abstract}
Behavioral stress has been shown to enhance long-term depression (LTD) in the CA1 region of the hippocampus, but the underlying mechanisms remain unclear. In the present study, we found that selectively blocking NR2B-containing NMDA receptors (NMDARs) abolishes the induction of LTD by prolonged low-frequency stimulation (LFS) in slices from stressed animals. Additionally, there is no need to activate NR2A-containing or synaptic NMDARs to induce this LTD, suggesting that LTD observed in slices from stressed animals is triggered primarily by extrasynaptic NMDAR activation. In contrast, stress has no effect on LTD induced by either a brief bath application of NMDA or a combination of LFS with the glutamate-uptake inhibitor DL-threo- $\beta$-benzyloxyaspartate (DL-TBOA). Furthermore, saturation of LFS-induced LTD in slices from stressed animals occludes the subsequent induction of LTD by LFS in the presence of DL-TBOA. We also found that stress induces a profound decrease in the glutamate uptake in the synaptosomal fraction of the hippocampal CA1 region. These effects were prevented when the animals were given a glucocorticoid receptor antagonist, $11 \beta, 17 \beta-11[4-$ (dimethylamino)phenyl]-17-hydroxy-17-(1-(propynyl)-estra-4,9-dien-3-one, before experiencing stress. These results suggest that the blockade of glutamate uptake is a potential mechanism underlying the stress-induced enhancement of LTD and point to a novel role for glutamate-uptake machinery in the regulation of synaptic plasticity induction.
\end{abstract}

Key words: stress; long-term depression; NMDA receptor; glutamate transporter; glucocorticoid receptor; hippocampus

\section{Introduction}

Long-term depression (LTD) is a persistent activity-dependent decrease in synaptic efficacy that, together with the converse process long-term potentiation, has been considered to be crucial for information storage in the brain (Malenka and Bear, 2004). Although LTD is very widely expressed at many locations in the brain, much of our understanding of its properties and functional relevance comes from studies on the mammalian hippocampus. In the hippocampus, LTD is divided into three categories: homosynaptic, heterosynaptic, and associative LTD (Linden and Connor, 1995). The best characterized form of homosynaptic LTD is induced in the CAl region of the hippocampus by prolonged low-frequency stimulation (LFS) $(0.5-3 \mathrm{~Hz})$ via an NMDA receptor (NMDAR)-dependent rise in postsynaptic intracellular $\mathrm{Ca}^{2+}$ and the activation of serine-threonine protein phosphatase cascades (Dudek and Bear, 1992; Mulkey et al., 1993, 1994). A cardinal feature of homosynaptic CA1 LTD is age dependence, because it is more robust in early postnatal life and declines with increasing age (Dudek and Bear, 1992; Wagner and Alger, 1995). A previous study failed to find the homosynaptic LTD induced by the LFS paradigm in naive slices from mature (5-10 weeks old) animals (Wagner and Alger, 1995). It remains unclear why LFS-

Received Jan. 31, 2005; revised March 21, 2005; accepted March 21, 2005

This work was supported by National Health Research Institute (Taipei, Taiwan) Research Grant NHRI-EX93-9215NI.

Correspondence should be addressed to Dr. Kuei-Sen Hsu, Department of Pharmacology, College of Medicine, National Cheng Kung University, Number 1, University Road, Tainan 701, Taiwan. E-mail: richard@mail.ncku.edu.tw. DOI:10.1523/JNEUROSCI.0406-05.2005

Copyright $\odot 2005$ Society for Neuroscience $\quad 0270-6474 / 05 / 254288-06 \$ 15.00 / 0$ induced LTD is not seen in the mature hippocampus. Interestingly, it has been shown that behavioral stress facilitates the induction of hippocampal CA1 LTD by LFS in the adult animals both in vivo and in vitro (Kim et al., 1996; Xu et al., 1997, 1998; Yang et al., 2004). However, to our knowledge, the cellular and molecular mechanism underlying the alteration of the inducibility of LTD by stress has not yet been studied.

In the present study, we report our novel observations that the facilitation of stress on subsequent LTD induction is mediated through the activation of glucocorticoid receptors, leading to the blockade of glutamate uptake and subsequently resulting in enhanced spillover of synaptically released glutamate by LFS acting on the extrasynaptic NR2B-containing NMDARs to undergo the induction of LTD.

\section{Materials and Methods}

Animals and stress protocol. Healthy adult male Sprague Dawley rats weighing 250-300 g were used. All procedures were performed according to National Institutes of Health guidelines for animal research (Guide for the Care and Use of Laboratory Animals) and approved by the Institutional Animal Care and Use Committee at National Cheng Kung University. Animals were housed in groups of four in a vivarum with a $12 \mathrm{~h}$ light/dark cycle, 50-60\% humidity, and ad libitum access to food and water. Animals were allowed to acclimate to the laboratory 1 week before the beginning of experiments. Behavioral stress was evoked by 60 tail shocks (1 mA for $1 \mathrm{~s} ; 30-90 \mathrm{~s}$ apart) while restrained in a Plexiglas tube. Blood samples were obtained by tail nick ( $300 \mu \mathrm{l}$ of blood was taken within 2 min after removal of the rats from the home cage) or decapitation (rats were killed within 3 min of being taken from the stress device) and immediately centrifuged at $1000 \times g$; plasma was separated and stored at $-20^{\circ} \mathrm{C}$. Plasma adrenocorticotropic hormone (ACTH) and 
corticosterone levels were determined by radioimmunoassay as described previously (Yang et al., 2004).

Preparations of hippocampal slice and electrophysiology. Promptly after stress, animals were killed, and hippocampal slices (400 $\mu \mathrm{m}$ thick) were prepared using standard procedures (Yang et al., 2004), allowed to recover for a minimum of $1 \mathrm{~h}$, and then transferred to a submersion-type recording chamber continually perfused with $30-32^{\circ} \mathrm{C}$ oxygenated artificial CSF solution containing the following (in $\mathrm{mm}$ ): $117 \mathrm{NaCl}, 4.7 \mathrm{KCl}$, $2.5 \mathrm{CaCl}_{2}, 1.2 \mathrm{MgCl}_{2}, 25 \mathrm{NaHCO}_{3}, 1.2 \mathrm{NaH}_{2} \mathrm{PO}_{4}$, and 11 glucose, $\mathrm{pH}$ 7.4. Extracellular recordings were performed with an Axoclamp 2B amplifier (Axon Instruments, Union City, CA). The responses were low-pass filtered at $2 \mathrm{kHz}$, digitally sampled at $5-10 \mathrm{kHz}$, and analyzed using pClamp software (version 8.0; Axon Instruments). The evoked postsynaptic responses were induced in CA1 stratum radiatum by stimulation of Schaffer collateral/commissural afferents at $0.033 \mathrm{~Hz}$ with a bipolar stimulating electrode. Field EPSPs (fEPSPs) were recorded with a glass pipette filled with $1 \mathrm{M} \mathrm{NaCl}$ (2-3 $\mathrm{M} \Omega$ resistance), and the initial slope was measured. LTD was induced using a standard protocol of 900 stimuli at $1 \mathrm{~Hz}$ (LFS).

Preparations of synaptosome and glutamate-uptake assay. The synaptosomal fractions were prepared from the CA1 region of hippocampal slices as described previously (Ortiz et al., 1995). In brief, the microdissected subregions were homogenized in $0.32 \mathrm{~m}$ sucrose, $1 \mathrm{~mm}$ EDTA, 4 $\mathrm{mm}$ Tris, and $10 \mathrm{~mm}$ glucose, $\mathrm{pH}$ 7.4, using a glass-Teflon homogenizer. Homogenates were centrifuged at $1000 \times g$ for $10 \mathrm{~min}\left(4^{\circ} \mathrm{C}\right)$. The resultant pellet was discarded, and the supernatant was spun at $9000 \times g$ for 10 $\min$ in a microcentrifuge at $4^{\circ} \mathrm{C}$. The pellets constituted the crude synaptosomal fraction. The crude fractions were resuspended in $1 \mathrm{ml}$ of HEPES buffer solution (in mM: $120 \mathrm{NaCl}, 4.7 \mathrm{KCl}, 2.2 \mathrm{CaCl}_{2}, 1.2 \mathrm{MgCl}_{2}$, 25 HEPES, $1.2 \mathrm{MgSO}_{4}, 1.2 \mathrm{KH}_{2} \mathrm{PO}_{4}$, and 10 glucose, $\mathrm{pH} 7.4$ ) to give a protein concentration of $0.5 \mathrm{mg} / \mathrm{ml}$, which was determined using a BioRad (Hercules, CA) Bradford protein assay kit. Glutamate-uptake assay was performed as described by Ullensvang et al. (1997), with minor modifications. Glutamate uptake of synaptosomes was initiated by adding $\left[{ }^{3} \mathrm{H}\right]$ glutamate $(10 \mathrm{~nm} ; 20-60 \mathrm{Ci} / \mathrm{mmol}$; Amersham Biosciences, Little Chalfont, UK) and $30 \mu \mathrm{M}$ unlabeled glutamate to the reaction tubes in a final volume of $500 \mu \mathrm{l}$ of HEPES buffer solution. After incubation at $37^{\circ} \mathrm{C}$ for $5 \mathrm{~min}$, the uptake was terminated by rapid filtration on glassfiber filters using a tissue harvester under vacuum, and the filter was washed five times with ice-cold HEPES buffer solution. Filters were dried overnight and countered on a liquid scintillation counter (Beckman Instruments, Fullerton, CA). Nonspecific uptake was determined with sodium-free solution that was prepared by replacing $\mathrm{NaCl}$ with choline chloride.

Materials. NMDA, D-APV, DL-threo- $\beta$-benzyloxyaspartate (DL-TBOA), $11 \beta, 17 \beta$-11[4-(dimethylamino)phenyl]-17-hydroxy-17-(1-(propynyl)estra-4,9-dien-3-one (RU38486), 3,3-oxo-7 propyl-17-hydroxy-androstan-4-en-17yl-propionic acid lactone (RU28318), (+)-5-methyl-10,11dihydroxy-5H-dibenzo(a,d)cyclohepten-5,10-imine [(+)-MK-801], and ifenprodil were obtained from Tocris Cookson (Bristol, UK). $R$-( $R, S)$ - $\alpha$-(4-hydroxyphenyl)- $\beta$-methyl-4-(phenylmethyl)-1-piperidine propranol (Ro25-6981) hydrochloride was purchased from Sigma (St. Louis, MO). [(R)-[(S)-1-(4-bromo-phenyl)-ethylamino]-(2,3dioxo-1,2,3,4-tetrahydro-quinoxalin-5-yl)-methyl]phosphonic acid (NVP-AAM077) was a generous gift from Novartis Pharma (Basel, Switzerland).

Data analysis. All data are expressed as mean \pm SEM, and the statistical significance was determined using the Mann-Whitney $U$ test or Student's $t$ test. The number of animals used is indicated by $n$. $p$ values $<$ 0.05 were considered to represent significant differences.

\section{Results}

Stress enhances LTD induction

In response to stress, significantly higher levels of ACTH (548 \pm 56 vs $102 \pm 18 \mathrm{pg} / \mathrm{ml}$ in basal condition; $p<0.05)$ and corticosterone concentrations $(119.6 \pm 6.8$ vs $9.2 \pm 2.1 \mathrm{ng} / \mathrm{ml}$ in basal condition; $p<0.05)$ were observed, confirming that our experimental restraint-tail-shock stressor activates hypothalamic-pi-
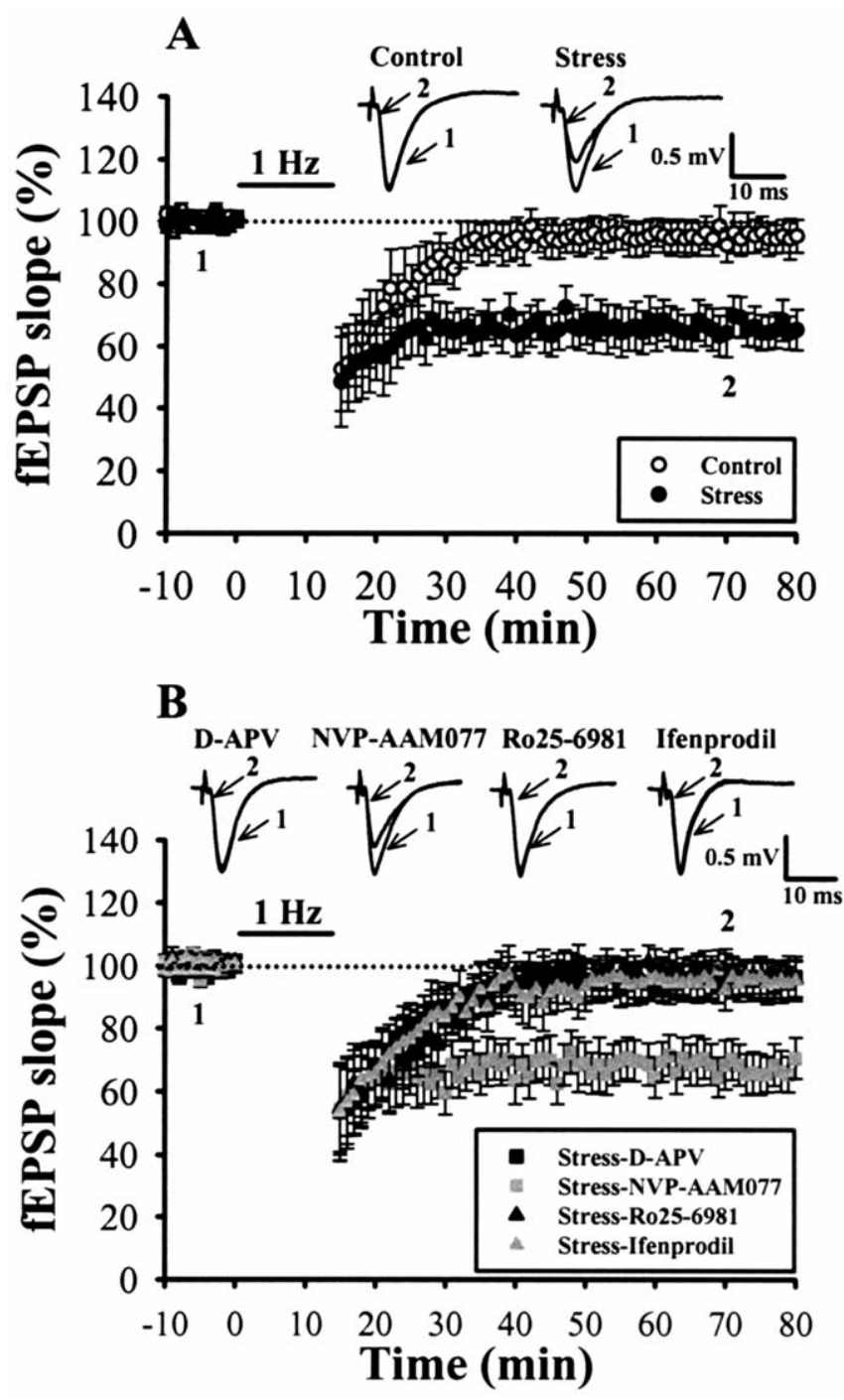

Figure 1. Stress facilitates subsequent LTD induction in the CA1 region of the hippocampus by NR2B-containing NMDAR activation. $A$, LFS induced robust LTD in slices from stressed rats. $\boldsymbol{B}$, LFS-induced LTD in slices from stressed rats was blocked by the nonselective NMDAR antagonist D-APV $(n=5)$ and the NR2B-selective antagonists Ro25-6981 $(n=5)$ and ifenprodil $(n=5)$ but not by the NR2A-selective antagonist NVP-AAM077 $(n=5)$. The superimposed fEPSPs in the top portion show respective recordings from example experiments taken at the time indicated by the number. Horizontal bars denote the period of the delivery of LFS. Dashed lines show the level of baseline.

tuitary-adrenal responses, as described previously (Kim et al., 1996; Yang et al., 2004). We next examined the effect of stress on the induction of LTD in the CA1 region of the hippocampus. In accordance with previous results (Kim et al., 1996; Yang et al., 2004), when LTD was assessed 50 min after the end of LFS, slices from stressed rats exhibited a robust LTD $(64.5 \pm 5.7 \%$ of baseline; $n=8$; $p<0.05)$ compared with slices from control rats $(95.3 \pm 5.3 \%$ of baseline; $n=8$ ) (Fig. $1 A$ ).

To assay the role of glucocorticoid receptor activation in mediating the enhancement of stress on LTD, the specific glucocorticoid receptor antagonist RU38486 (40 mg/kg) was injected intraperitoneally $30 \mathrm{~min}$ before stress. As shown in supplemental Figure $1 \mathrm{~A}$ (available at www.jneurosci.org as supplemental material), administration of RU38486 completely prevented the stress-induced enhancement $(93.5 \pm 4.5 \%$ of baseline; $n=5)$. Conversely, previous administration of the specific mineralocor- 
ticoid receptor antagonist RU28318 $(10 \mathrm{mg} / \mathrm{kg})$ did not affect the stress effect ( $71.5 \pm 6.3 \%$ of baseline; $n=5$ ) (supplemental Fig. $1 B$, available at www.jneurosci.org as supplemental material).

\section{LTD observed in slices from stressed rats relies on} NR2B-containing NMDARs

It is well established that activation of NMDARs is required for LFS-induced LTD in the hippocampal CA1 neurons (Dudek and Bear, 1992; Mulkey and Malenka, 1992). We thus examined whether LTD observed in slices from stressed rats is also NMDAR dependent. We found that blocking of NMDARs by perfusing D-APV $(50 \mu \mathrm{M})$ completely prevented the induction of LTD by LFS ( $94.8 \pm 5.7 \%$ of baseline; $n=5 ; p<0.05$ when compared with the control stressed slices) (Fig. $1 B$ ). NMDARs are heteromers composed of two essential NR1 subunits and two or three NR2 subunits (Seeburg, 1993; Cull-Candy et al., 2001). There are four NR2 subunits (NR2A-NR2D), but the NR2A and NR2B subunits predominate in the adult rat hippocampus (Laurie et al., 1997; Wenzel et al., 1997). Because the presence of different NR2 subunits may confer distinct gating and pharmacological properties to heteromeric NMDARs (Monyer et al., 1994) and couple them to different cytoplasmic signaling cascades (Sala et al., 2000; Kohr et al., 2003), we next discerned which NMDAR subpopulations are required for the induction of LTD by LFS in slices from stressed rats. We first investigated the effect of blocking NR2Acontaining NMDARs on the induction of LTD. Preincubation of the slices with the NR2A-selective antagonist NVP-AAM077 (0.5 $\mu \mathrm{M})$ (Auberson et al., 2002) did not prevent the LFS-induced LTD $(68.4 \pm 6.5 \%$ of baseline; $n=5 ; p>0.05$ when compared with the control stressed slices) (Fig. $1 B$ ). In contrast, in the presence of the NR2B-selective antagonists Ro25-6981 (0.5 $\mu \mathrm{M})$ or ifenprodil (3 $\mu \mathrm{M})$, LFS failed to induce LTD (Ro25-6981, $96.5 \pm 6.5 \%$ of baseline, $n=5$; ifenprodil, $95.3 \pm 5.2 \%$ of baseline, $n=5 ; p<0.05$ when compared with the control stressed slices) (Fig. $1 B$ ). These results suggest that the induction of LTD by LFS in stressed slices relies on the activation of NR2Bcontaining NMDARs.

Because there is good electrophysiological evidence that NR2B-containing NMDARs appeared predominantly at extrasynaptic sites of mature synapses (Stocca and Vicini, 1998; Rumbaugh and Vicini, 1999; Tovar and Westbrook, 1999), we were curious to determine whether the induction of LTD by LFS in slices from stressed rats relies on the activation of extrasynaptic NMDARs. To examine this question, we took advantage of the fact that the application of NMDA to hippocampal slices exclusively causes LTD via the activation of extrasynaptic NMDARs (Lee et al., 1998). We found that a 3 min application of $20 \mu \mathrm{M}$ NMDA induced a robust LTD in slices from both control and stressed rats (Fig. 2A). On average, the slope of fEPSP measured 50 min after washout of NMDA was to $54.8 \pm 5.5 \%$ of baseline $(n=6)$ in slices from stressed rats, which was not significantly different from that of LTD recorded in slices from control rats $(63.5 \pm 5.2 \%$ of baseline; $n=5)$.

Considering that NMDA-induced LTD could be attributable to the preferential activation of extrasynaptic or nonselective activation of both synaptic and extrasynaptic NMDARs, we therefore tested whether the activation of extrasynaptic NMDARs alone is sufficient for the induction of LTD by LFS observed in slices from stressed rats. To this end, LFS-induced LTD was examined when synaptic NMDARs were selectively blocked by pretreating slices with MK-801 (5 $\mu \mathrm{M})$ for $30 \mathrm{~min}$ (MacDonald and Nowak, 1990; Rosenmund et al., 1993). To subsequently activate extrasynaptic NMDARs, MK-801 was washed out for $60 \mathrm{~min}$, LFS
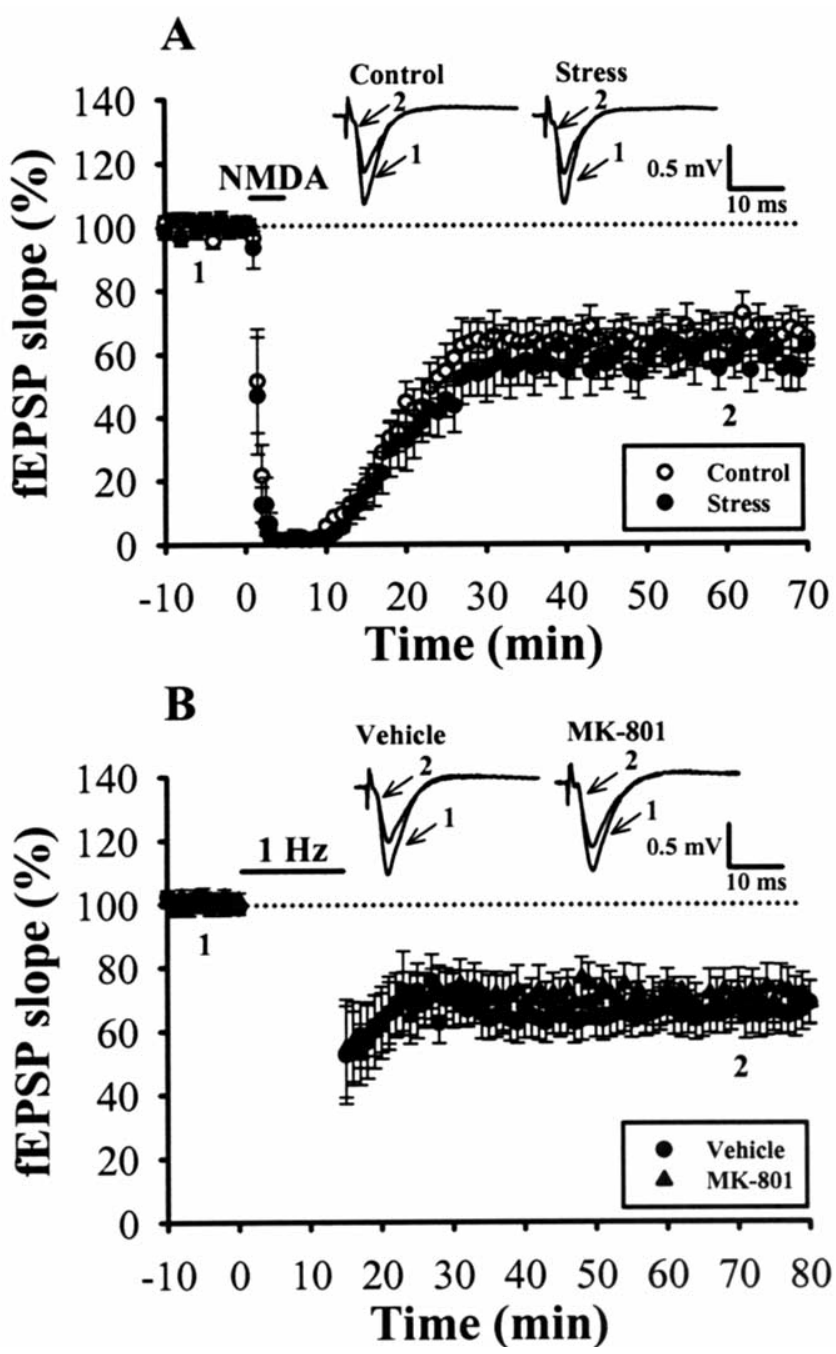

Figure 2. Extrasynaptic NMDARs mediate LTD in slices from stressed rats. $\boldsymbol{A}$, Summary of experiments showing that a brief application of NMDA $(20 \mu \mathrm{M})$ for 3 min produced an LTD of fEPSPs in slices from both control $(n=5)$ and stressed $(n=6)$ rats. $B$, The induction of LTD by LFS was not significantly affected when synaptic NMDARs were selectively blocked by pretreating slices with MK-801 for $30 \mathrm{~min}$.

was delivered in slices from stressed rats, and the magnitude of LTD was examined. Under this condition, LFS still resulted in the induction of significant LTD (71.5 $\pm 6.6 \%$ of baseline; $n=5)$, which was not significantly different from that of LTD recorded in slices from vehicle pretreatment $(0.1 \%$ DMSO; $64.5 \pm 6.5 \%$ of baseline; $n=5$ ) (Fig. $2 B$ ). These results suggest that the activation of extrasynaptic NMDARs alone is sufficient to induce LTD in slices from stressed rats.

\section{Spillover and temporal summation of glutamate allows}

\section{LFS-induced LTD}

Recent study has shown that LFS is able to induce LTD in adult cortex after inhibition of glutamate uptake and that this form of LTD relies on the activation of extrasynaptic NR2B-containing NMDARs (Massey et al., 2004). We next wanted to determine whether this mechanism could provide a possible explanation for why LFS can induce a robust LTD in slices from stressed rats. The best approach to address this question is to determine whether saturation of one form of LTD occludes the induction of the other form at the same synapses. We first examined the effect of blocking glutamate uptake on the inducibility of LTD in the CA1 
region of adult rat hippocampal slices. In accordance with previous results (Massey et al., 2004), we found that LFS induced a robust LTD in the presence of the glutamate-uptake inhibitor DL-TBOA $(10 \mu \mathrm{M})$ in slices from both control and stressed rats (Fig. 3A). On average, the slope of fEPSP measured $50 \mathrm{~min}$ after LFS was to $58.5 \pm 5.5 \%$ of baseline $(n=5)$ in slices from stressed rats, which was not significantly different from that of LTD recorded in slices from control rats $(71.5 \pm 5.7 \%$ of baseline; $n=$ $5)$. Although the slices from stressed rats showed a higher level of LTD than that produced by the same stimulation in slices from control rats, it did not reach statistical significance $(p=0.12)$. In slices from stressed rats, after LFS-induced LTD was fully established, no additional LTD was induced by LFS in the presence of DL-TBOA $(96.2 \pm 6.9 \%$ of renormalized baseline, measured 50 min after LFS; $n=6$ ) (Fig. $3 B, C$ ). Such an occlusion experiment demonstrates that these two forms of LTD may share some common mechanisms.

\section{Stress impairs glutamate uptake}

The preceding results point out that the facilitation of LTD induction by stress may arise from the blockade of glutamate uptake, whereby synaptically released glutamate by LFS could activate extrasynaptic NMDARs. To test the potential changes in the glutamate-uptake machinery after stress, the crude synaptosomal fractions of the hippocampal CA1 region were prepared for glutamate-uptake assay. We found that the glutamate uptake was markedly decreased in the synaptosomes isolated from stressed rats (Fig. 4). This impairment was prevented when the animals were given a glucocorticoid receptor antagonist, RU38486, before experiencing stress.

\section{Discussion}

Previous findings demonstrated that the induction of LTD in adult animals is facilitated by behavioral stress via an unknown mechanism (Kim et al., 1996; Xu et al., 1997, 1998; Yang et al., 2004). This study provides the electrophysiological and pharmacological evidence describing the potential basis of the stress-induced enhancement of LTD. Our findings demonstrate that stress increases the circulating corticosterone levels, leading to the activation of glucocorticoid receptors and subsequently inhibiting the uptake of synaptically released glutamate. Accordingly, the spillover and temporal summation of synaptically released glutamate by LFS would activate extrasynaptic NR2B-containing NMDARs to undergo the induction of LTD in adult hippocampal CA1 region.

It has been shown previously that stress facilitates the induction of LTD through NMDAR activation (Kim et al., 1996). Consistent with this finding, our data showed that D-APV completely prevented the induction of LTD by LFS in slices from stressed rats. In addition, we extended this finding by demonstrating that the NR2B-selective antagonists Ro25-6981 and ifenprodil specifically blocked the induction of LTD, whereas an NR2A-selective antagonist, NVP-AAM077, had no significant effect on LTD. This suggests that the activation of NR2B-containing NMDARs is specifically involved in LFS-induced LTD in stressed adult animals. Because it is not yet known whether NVP-AAM077 acts at NR1/NR2A/NR2B triheteromers, we cannot exclude the possibility that such heteromers are involved in stress-induced enhancement of LTD. Our finding is consistent with the current observation suggesting a role for NR2B-containing NMDARs in hippocampal CA1 LTD induction in young adult rats (Liu et al., 2004). Similarly, Massey et al. (2004) showed, using glutamateuptake inhibitors, that LFS-induced a de novo LTD in adult cortex
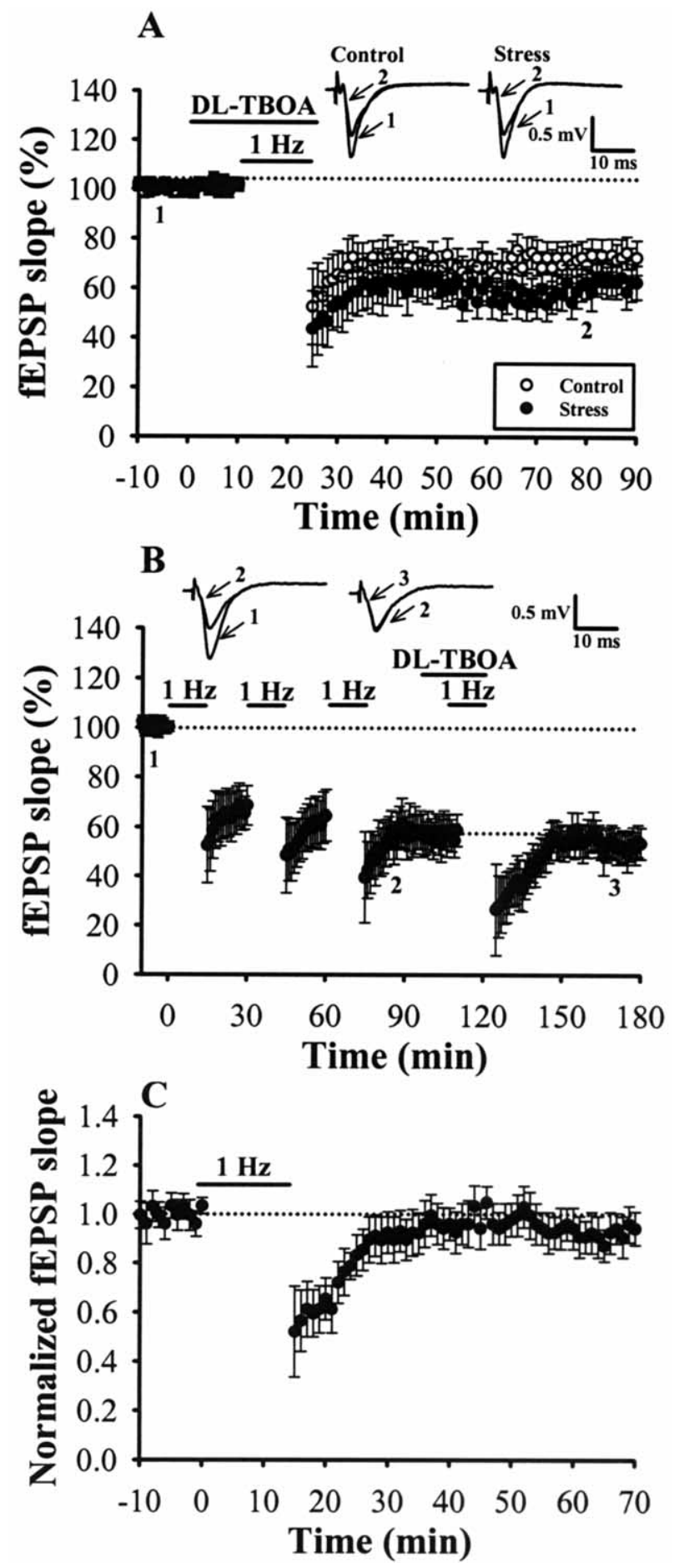

Figure 3. Saturation of LFS-induced LTD occludes LFS-induced LTD in the presence of glutamate-uptake inhibitor. $A$, Summary of experiments showing that LFS in the presence of DL-TBOA resulted in the induction of LTD in slices from both control $(n=5)$ and stressed $(n=5)$ rats. $\boldsymbol{B}$, Summary of four experiments showing that, in slices from stressed rats, after LFSinduced LTD was fully established ( 3 episodes of $1 \mathrm{~Hz}$ for 15 min stimuli), LFS in the presence of DL-TBOA failed to induce additional synaptic depression $(n=6)$. $\boldsymbol{C}$, Data taken from $\boldsymbol{B}$, renormalized to the 10 min period preceding the application of LFS. Horizontal bars denote the period of the delivery of LFS or DL-TBOA.

that is also dependent on the activation of NR2B-containing NMDARs.

Two lines of evidence suggest that stress facilitates the induction of LTD through the activation of extrasynaptic NMDARs. 


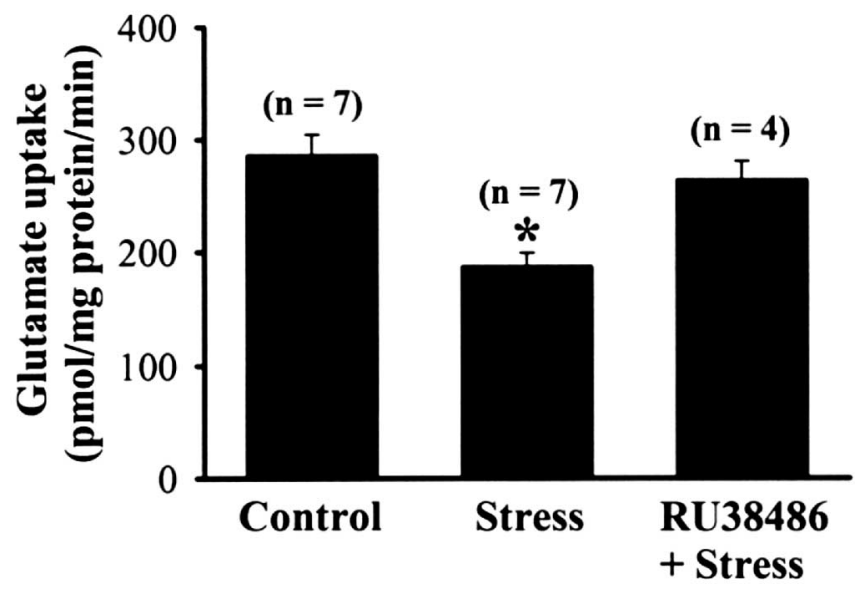

Figure 4. Stress impairs glutamate uptake in hippocampal CA1 synaptosomes. Administration of the glucocorticoid receptor antagonist RU38486 completely prevented the inhibition of stress on glutamate uptake. The results were presented as mean \pm SEM of four to seven rats per group. ${ }^{*} p<0.05$ compared with the control group.

First, the magnitude of LFS-induced LTD in slices from stressed rats was not significantly affected by pretreating slices with MK801 (Fig. 2 B). Second, saturation of LFS-induced LTD can occlude the subsequent induction of LTD by LFS in the presence of glutamate-uptake inhibitor (Fig. 3B), suggesting overlapping mechanisms. In fact, previous studies have demonstrated that blocking glutamate uptake may result in the induction of LTD by allowing glutamate released by LFS to activate extrasynaptic NMDARs (Chen and Diamond, 2002; Clark and Cull-Candy, 2002; Massey et al., 2004). A question arises as to how the glutamate released by LFS might activate extrasynaptic NMDARs to induce LTD in slices from stressed rats. It is generally thought that, under normal conditions, the synaptically released glutamate is short lived and is unlikely to be sufficient to activate extrasynaptic receptors. The inclusion of extrasynaptic NMDARs in a synaptic event appears to vary according to several parameters, including the location and degree of saturation of neuronal and astroglial transporters, the activity of transporters, and the amount, timing, and proximity of glutamate release (van Zundert et al., 2004). Thus, an intriguing possibility is that stress causes a decrease in glutamate uptake, which results in the spillover and temporal summation of synaptically released glutamate by LFS to activate extrasynaptic NMDARs. This suggestion is supported by the fact that glutamate uptake in isolated hippocampal CA1 synaptosomes was significantly decreased after stress (Fig. 4).

The mechanisms by which stress impairs glutamate-uptake systems remain to be determined. Previous work from our laboratory has demonstrated that stress can increase the PKC activity in the hippocampal CA1 neurons (Yang et al., 2004). In addition, it has been shown that PKC activation inhibited the GLAST (glutamate-aspartate transporter) activity via direct phosphorylation (Conradt and Stoffel, 1997; Bernabe et al., 2003). Thus, it seems possible that the reduction of glutamate uptake after stress is involved in an increased PKC-mediated phosphorylation of glutamate transporters, in turn leading to an inactivation of the transporters, but additional studies will be necessary to examine this issue.

In conclusion, the present study provides a mechanism to explain the stress-induced facilitation of LTD induction and unveils a novel relationship between glutamate-uptake machinery and LTD. Considering that stress-facilitated LTD may induce output plasticity through synchronized spikes and spontaneous unitary discharges to other brain structures under stress conditions and thus may contribute to the permanent storage of the information of stress events (Cao et al., 2004), our findings may also provide new opportunities for the development of more selective medication that targets these pathways and prevents their malfunction.

\section{References}

Auberson YP, Allgeier H, Bischoff S, Lingenhoehl K, Moretti R, Schmutz M (2002) 5-Phosphonomethylquinoxalinediones as competitive NMDA receptor antagonists with a preference for the human $1 \mathrm{~A} / 2 \mathrm{~A}$, rather than 1A/2B receptor composition. Bioorg Med Chem Lett 12:1099-1102.

Bernabe A, Mendez JA, Hernandez-Kelly LC, Ortega A (2003) Regulation of the $\mathrm{Na}^{+}$-dependent glutamate/aspartate transporter in rodent cerebellar astrocytes. Neurochem Res 28:1843-1849.

Cao J, Chen N, Xu T, Xu L (2004) Stress-facilitated LTD induces output plasticity through synchronized-spikes and spontaneous unitary discharges in the CA1 region of the hippocampus. Neurosci Res 49:229-239.

Chen S, Diamond JS (2002) Synaptically released glutamate activates extrasynaptic NMDA receptors on cells in the ganglion cell layer of rat retina. J Neurosci 22:2165-2173.

Clark BA, Cull-Candy SG (2002) Activity-dependent recruitment of extrasynaptic NMDA receptor activation at an AMPA receptor-only synapse. J Neurosci 22:4428-4436.

Conradt M, Stoffel W (1997) Inhibition of the high-affinity brain glutamate transporter GLAST-1 via direct phosphorylation. J Neurochem 68:1244-1251.

Cull-Candy S, Brickley S, Farrant M (2001) NMDA receptor subunits: diversity, development and disease. Curr Opin Neurobiol 11:327-335.

Dudek SM, Bear MF (1992) Homosynaptic long-term depression in area $\mathrm{CA} 1$ of hippocampus and effects of $\mathrm{N}$-methyl-D-aspartate receptor blockade. Proc Natl Acad Sci USA 89:4363-4367.

Kim JJ, Foy MR, Thompson RF (1996) Behavioral stress modifies hippocampal plasticity through $N$-methyl-D-aspartate receptor activation. Proc Natl Acad Sci USA 93:4750-4753.

Kohr G, Jensen V, Koester HJ, Mihaljevic AL, Utvik JK, Kvello A, Ottersen OP, Seeburg PH, Sprengel R, Hvalby O (2003) Intracellular domains of NMDA receptor subtypes are determinants for long-term potentiation induction. J Neurosci 23:10791-10799.

Laurie DJ, Bartke I, Schoepfer R, Naujoks K, Seeburg PH (1997) Regional, developmental and interspecies expression of the four NMDAR2 subunits, examined using monoclonal antibodies. Brain Res Mol Brain Res 51:23-32.

Lee HK, Kameyama K, Huganir RL, Bear MF (1998) NMDA induces longterm synaptic depression and dephosphorylation of the GluR1 subunit of AMPA receptors in hippocampus. Neuron 21:1151-1162.

Linden DJ, Connor JA (1995) Long-term synaptic depression. Annu Rev Neurosci 18:319-357.

Liu L, Wong TP, Pozza MF, Lingenhoehl K, Wang Y, Sheng M, Auberson YP, Wang YT (2004) Role of NMDA receptor subtypes in governing the direction of hippocampal synaptic plasticity. Science 304:1021-1024.

MacDonald JF, Nowak LM (1990) Mechanisms of blockade of excitatory amino acid receptor channels. Trends Pharmacol Sci 11:167-172.

Malenka RC, Bear MF (2004) LTP and LTD: an embarrassment of riches. Neuron 44:5-21.

Massey PV, Johnson BE, Moult PR, Auberson YP, Brown MW, Molnar E, Collingridge GL, Bashir ZI (2004) Differential roles of NR2A and NR2B-containing NMDA receptors in cortical long-term potentiation and long-term depression. J Neurosci 24:7821-7828.

Monyer H, Burnashev N, Laurie DJ, Sakmann B, Seeburg PH (1994) Developmental and regional expression in the rat brain and functional properties of four NMDA receptors. Neuron 12:529-540.

Mulkey RM, Malenka RC (1992) Mechanisms underlying induction of homosynaptic long-term depression in area CA1 of the hippocampus. Neuron 9:967-975.

Mulkey RM, Herron CE, Malenka RC (1993) An essential role for protein phosphatases in hippocampal long-term depression. Science 261:1051-1055.

Mulkey RM, Endo S, Shenolikar S, Malenka RC (1994) Involvement of a calcineurin/inhibitor-1 phosphatase cascade in hippocampal long-term depression. Nature 369:486-488. 
Ortiz J, Harris HW, Guitart X, Terwilliger RZ, Haycock JW, Nestler EJ (1995) Extracellular signal-regulated protein kinases (ERKs) and ERK kinase (MEK) in brain: regional distribution and regulation by chronic morphine. J Neurosci 15:1285-1297.

Rosenmund C, Clements JD, Westbrook GL (1993) Nonuniform probability of glutamate release at a hippocampal synapse. Science 262:754-757.

Rumbaugh G, Vicini S (1999) Distinct synaptic and extrasynaptic NMDA receptors in developing cerebellar granule neurons. J Neurosci 19:10603-10610.

Sala C, Rudolph-Correia S, Sheng M (2000) Developmentally regulated NMDA receptor-dependent dephosphorylation of cAMP response element-binding protein (CREB) in hippocampal neurons. J Neurosci 20:3529-3536.

Seeburg PH (1993) The TINS/TiPS lecture. The molecular biology of mammalian glutamate receptor channels. Trends Neurosci 16:359-365.

Stocca G, Vicini S (1998) Increased contribution of NR2A subunit to synaptic NMDA receptors in developing rat cortical neurons. J Physiol (Lond) 507:13-24.

Tovar KR, Westbrook GL (1999) The incorporation of NMDA receptors with a distinct subunit composition at nascent hippocampal synapses in vitro. J Neurosci 19:4180-4188.

Ullensvang K, Lehre KP, Storm-Mathisen J, Danbolt NC (1997) Differential developmental expression of the two rat brain glutamate transporter proteins GLAST and GLT. Eur J Neurosci 9:1646-1655.

van Zundert B, Yoshii A, Constantine-Paton M (2004) Receptor compartmentalization and trafficking at glutamate synapses: a developmental proposal. Trends Neurosci 27:428-437.

Wagner JJ, Alger BE (1995) GABAergic and developmental influences on homosynaptic LTD and depotentiation in rat hippocampus. J Neurosci 15:1577-1586.

Wenzel A, Fritschy JM, Mohler H, Benke D (1997) NMDA receptor heterogeneity during postnatal development of the rat brain: differential expression of the NR2A, NR2B, and NR2C subunit proteins. J Neurochem 68:469-478.

Yang CH, Huang CC, Hsu KS (2004) Behavioral stress modifies hippocampal synaptic plasticity through corticosterone-induced sustained extracellular signal-regulated kinase/mitogen-activated protein kinase activation. J Neurosci 24:11029-11034.

Xu L, Anwyl R, Rowan MJ (1997) Behavioural stress facilitates the induction of long-term depression in the hippocampus. Nature 387:497-500.

Xu L, Holscher C, Anwyl R, Rowan MJ (1998) Glucocorticoid receptor and protein/RNA synthesis-dependent mechanisms underlie the control of synaptic plasticity by stress. Proc Natl Acad Sci USA 95:3204-3208. 